Letter to the Editor

\section{Do reductions in ghrelin contribute towards antipsychotic-induced weight gain?}

Keywords:

Psychosis

Antipsychotic

Obesity

Metabolic

Cardiovascular

Nutrition

We read with interest the recent meta-analysis by Goetz and Miller (2018), which reported that circulating levels of ghrelin are significantly reduced by olanzapine treatment. This finding may shed new light on the behavioural and metabolic side-effects of olanzapine, as ghrelin is widely referred to as 'the hunger hormone', due to its well-established role in appetite regulation and satiety (Higgins et al., 2007). Somewhat paradoxically, reductions in ghrelin levels are an established risk factor for both binge-eating (Geliebter et al., 2005) and obesity (Tschöp et al., 2001). Indeed, previous research has suggested the drastic weight gain observed in with second-generation antipsychotic treatment may be mediated by increases in appetite (Mayfield et al., 2016). Supporting this, recent population-scale studies have found that individuals with psychotic disorders are not only less active than the general population (Firth et al., 2018b), but also consume substantially more total calories and obesogenic foods (i.e. sugars, carbohydrates, and saturated fats) than people with other mental disorders, even after adjusting for social deprivation and education (Firth et al., 2018a).

Thus, olanzapine's ghrelin-reducing effects, demonstrated by Goetz and Miller (2018), could be partially responsible for the weight gain which arises in those taking this medication. However, Goetz and Miller (2018) did not examine how olanzapine-induced changes in ghrelin may be associated with changes in bodyweight. Thus, we aimed to build on these initial findings, using meta-regression analyses, and a review of the individual studies, to investigate the link between olanzapine's effects on ghrelin and subsequent weight gain.

All analyses were conducted in Comprehensive Meta-Analysis V3. For study characteristics and effects of olanzapine on ghrelin levels, we re-used the individual study data and standardized mean differences (SMDs) presented by Goetz and Miller (2018). Following this, we returned to the original studies to calculate SMDs for the effects of olanzapine on BMI (or using bodyweight changes as a proxy, where BMI itself was not reported) for each study. We then conducted metaregression analyses to examine the extent to which effects on BMI following olanzapine treatment were related to the effects of olanzapine on ghrelin.

Five of the six studies from Goetz and Miller (2018) were included in our analysis (as Smith et al. (2012), did not report changes in BMI). First, a random effect meta-analysis confirmed a significant increase in BMI following olanzapine treatment $(N=5, n=98, \mathrm{SMD}=0.412,95 \%$.CI $=0.003$ to $0.821, p=0.048$ ). Following this, our meta-regression analyses found that weight gain from olanzapine was associated with reductions in ghrelin. As shown in Fig. 1; increases in BMI following olanzapine treatment held a significant inverse relationship with the effect of olanzapine on circulating ghrelin levels $(B=-0.877$, S.E. $=$ $0.363, p=0.016$ ). This relationship also persisted when adjusting for study length (in weeks) $(B=-0.950$, S.E. $=0.229, p \leq 0.001)$. Thus, the analyses show that greater reductions in ghrelin from olanzapine treatment are accompanied by larger amounts of olanzapine-induced weight gain.

Only two individual studies examined associations within their samples between changes in ghrelin and changes in bodyweight/BMI. The weak correlation in Basoglu et al. (2010) did not reach statistical significance ( $r=-0.212, p>0.05$ ), potentially due to short duration of study (6 weeks). Nonetheless, the six-month study by Kim et al. (2008) did observe a significant relationship between ghrelin reductions and olanzapine-induced weight gain $(r=-0.411, p=0.046)$. Pooling the two correlations together found that ghrelin reductions following olanzapine treatment were significantly associated with weight gain ( $n=42$, Fisher's $Z=-0.345$, S.E. $=0.167, p=0.039$ ).

Overall, the results of our meta-regression, the individual studies, and Goetz and Miller (2018)'s meta-analysis find consistent support for the hypothesis that obesogenic effects of olanzapine are partly attributable to its effects on ghrelin. The number of studies on this topic is currently too small for any definitive analyses, and so the result of our meta-regression can only be viewed as preliminary - although strongly indicative of a clear rationale for further research. Specifically, large-scale, robust studies are now required to establish the extent to which antipsychotic-induced weight gain is driven by effects on ghrelin, and how this differs across agents.

Clinically, the findings presented here contribute to the growing evidence that the obesity and metabolic dysfunction observed in schizophrenia is partly driven by appetite-increasing side effects of SGAs; underpinned by biological changes in appetite control. This further adds to the idea that providing dietary interventions and healthy lifestyle programs should be considered as a 'duty of care' when initiating certain medications (e.g. olanzapine/clozapine) to people with mental illness. A wealth of research in the general population shows that preventing weight gain is more efficient than attempting to reverse long-term obesity. Thus, the rapidity of the hormonal and metabolic changes induced by olanzapine suggests that lifestyle interventions should commenced at time of initiation of SGA treatment, rather than as a response to significant weight-gain, in order to maintain physical health from the outset.

\section{Conflict of interests}

JF is supported by a Blackmores Institute Fellowship. ST is funded by the South Eastern Sydney Local Health District in a clinical position. JS has received either presentation honoraria, travel support, clinical trial grants, book royalties, or independent consultancy payments from: Integria Healthcare \& MediHerb, Pfizer, Scius Health, Key 


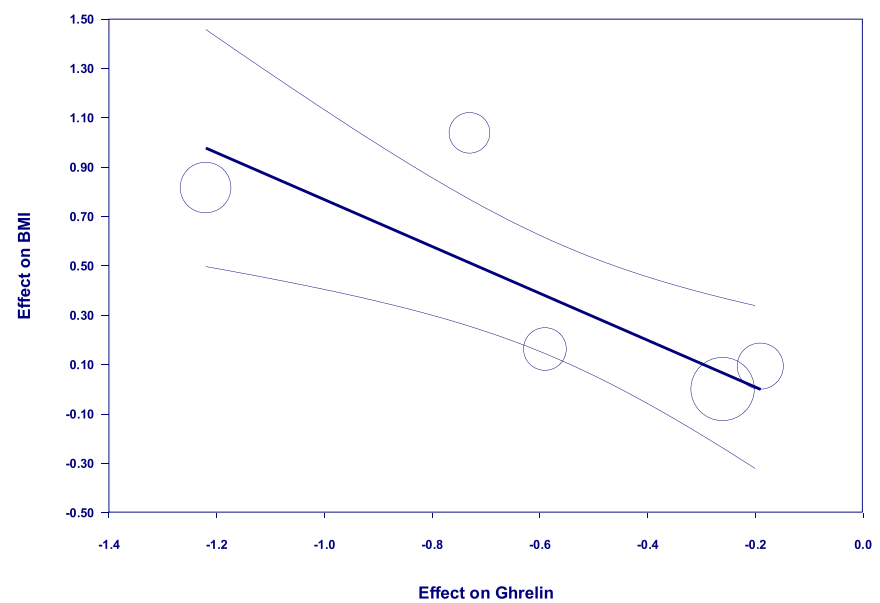

Fig. 1. Meta-regression showing the relationship between olanzapine's effects on both ghrelin and BMI. Circle size represents individual study weighting. Central line represents line of best fit, with 95\% confidence intervals.

Pharmaceuticals, Taki Mai, Bioceuticals \& Blackmores, Soho-Flordis, Healthworld, HealthEd, HealthMasters, Kantar Consulting, Research Reviews, Elsevier, Chaminade University, International Society for Affective Disorders, Complementary Medicines Australia, SPRIM, Terry White Chemists, ANS, Society for Medicinal Plant and Natural Product Research, Sanofi-Aventis, Omega-3 Centre, the National Health and Medical Research Council, CR Roper Fellowship. All other authors have nothing to declare.

\section{Contributors}

JF conducted the analyses and prepared initial draft of manuscript. ST assisted in data extraction and analysis under the mentorship of JF. SEJ, JS, MS, DS, DV were all integral in the intellectual conception, execution and writing and revising of the paper. All authors have approved the final manuscript.

\section{Acknowledgements}

JF is supported by a Blackmores Institute Fellowship. JS is funded by an NHMRC Research Fellowship (APP1125000). DS is supported in part by an NHMRC ECF APP1111136.

\section{References}

Basoglu, C., Oner, O., Gunes, C., Semiz, U.B., Ates, A.M., Algul, A., et al., 2010. Plasma orexin A, ghrelin, cholecystokinin, visfatin, leptin and agouti-related protein levels during 6week olanzapine treatment in first-episode male patients with psychosis. Int. Clin. Psychopharmacol. 25, 165-171.

Firth, J., Stubbs, B., Teasdale, S.B., Ward, P.B., Veronese, N., Shivappa, N., et al., 2018a. Diet as a hot topic in psychiatry: a population-scale study of nutritional intake and inflammatory potential in severe mental illness. World Psychiatry 17, 365-367.

Firth, J., Stubbs, B., Vancampfort, D., Schuch, F.B., Rosenbaum, S., Ward, P.B., et al., 2018b. The validity and value of self-reported physical activity and accelerometry in people with schizophrenia: a population-scale study of the UK biobank. Schizophr. Bull. 44, 1293-1300.

Geliebter, A., Gluck, M.E., Hashim, S.A., 2005. Plasma ghrelin concentrations are lower in binge-eating disorder. J. Nutr. 135, 1326-1330.

Goetz, R.L., Miller, B.J., 2018. Meta-analysis of ghrelin alterations in schizophrenia: effects of olanzapine. Schizophr. Res. https://doi.org/10.1016/j.schres.2018.11.036.
Higgins, S.C., Gueorguiev, M., Korbonits, M., 2007. Ghrelin, the peripheral hunger hormone. Ann. Med. 39, 116-136.

Kim, B.-J., Sohn, J.-W., Park, C.-S., Hahn, G.-H., Koo, J., Noh, Y.-D., et al., 2008. Body weight and plasma levels of ghrelin and leptin during treatment with olanzapine. J. Korean Med. Sci. 23, 685-690.

Mayfield, K., Siskind, D., Winckel, K., Russell, A.W., Kisely, S., Smith, G., et al., 2016 Glucagon-like peptide-1 agonists combating clozapine-associated obesity and diabetes. J. Psychopharmacol. (Oxf.) 30, 227-236.

Smith, R.C., Rachakonda, S., Dwivedi, S., Davis, J.M., 2012. Olanzapine and risperidone effects on appetite and ghrelin in chronic schizophrenic patients. Psychiatry Res. 199, 159-163.

Tschöp, M., Weyer, C., Tataranni, P.A., Devanarayan, V., Ravussin, E., Heiman, M.L., 2001 Circulating ghrelin levels are decreased in human obesity. Diabetes 50, 707-709.

Joseph Firth*

NICM Health Research Institute, Western Sydney University, Westmead,

Australia

Division of Psychology and Mental Health, Faculty of Biology, Medicine and Health, University of Manchester, UK

Centre for Youth Mental Health, University of Melbourne, Melbourne,

Australia

${ }^{*}$ Corresponding author at: NICM Health Research Unit, Western Sydney University, Westmead, Australia.

E-mail address: j.firth@westernsydney.edu.au.

Scott B. Teasdale

School of Psychiatry, Faculty of Medicine, UNSW, Sydney, Australia Keeping the Body in Mind Program, South Eastern Sydney Local Health

District, Sydney, Australia

Sarah E. Jackson

Department of Behavioural Science and Health, University College London,

London, England, UK

Davy Vancampfort

KU Leuven, Department of Rehabilitation Sciences, University Psychiatric Center, Kortenberg, Belgium

Dan Siskind

University of Queensland School of Medicine, Brisbane, Qld, Australia Metro South Addiction and Mental Health Service, Brisbane, Qld, Australia

Jerome Sarris

NICM Health Research Institute, Western Sydney University, Westmead,

Australia

Department of Psychiatry, University of Melbourne, Professorial Unit, The Melbourne Clinic, Melbourne, Australia

Marco Solmi

University of Padua, Neurosciences Department, Padua, Italy Padua University Hospital, Psychiatry Unit, Padua, Italy

21 December 2018 Available online $\mathrm{xxxx}$ 